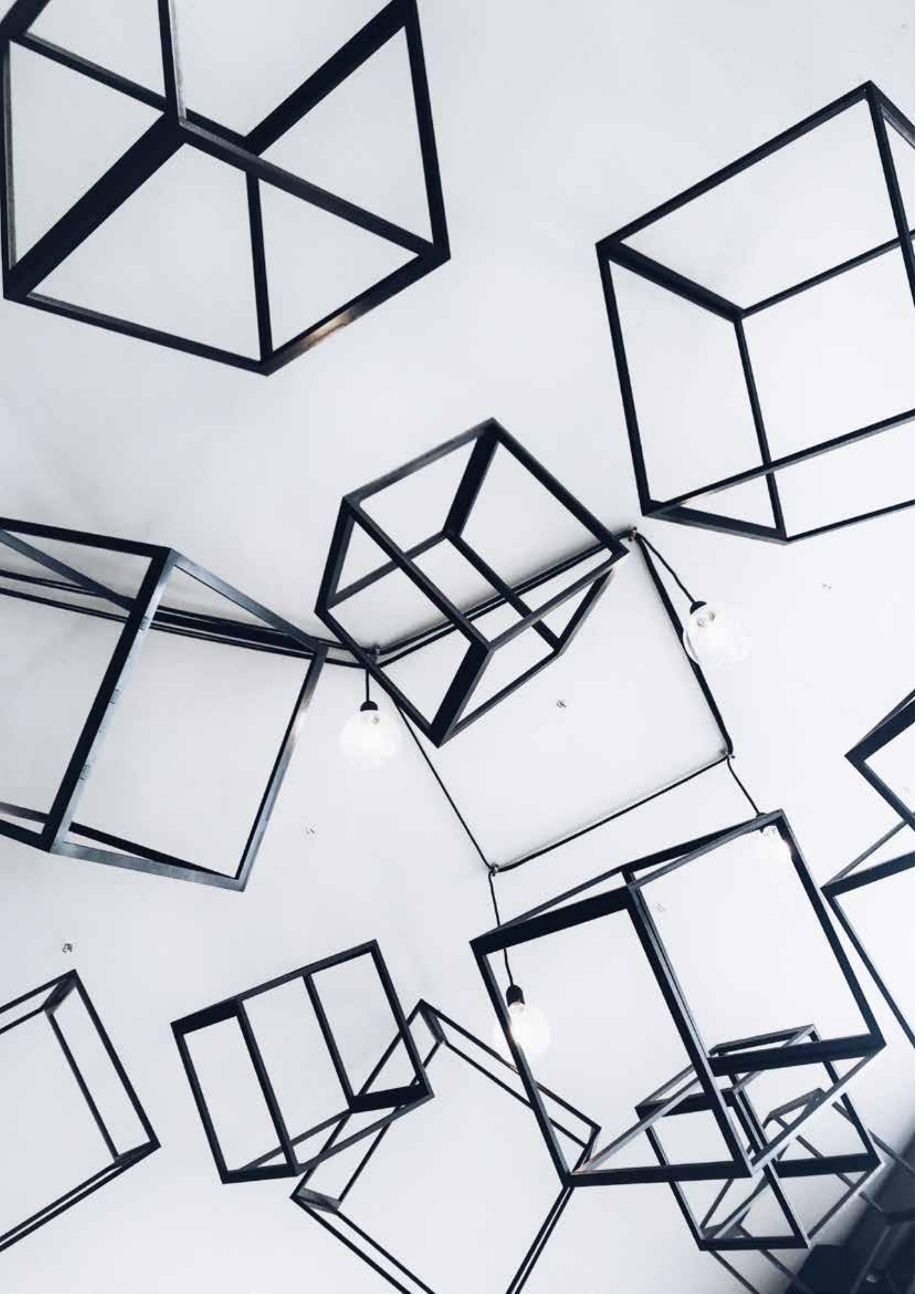

\title{
Altas capacidades: qué conocen los docentes sobre este alumnado
}

Carolina Seade-Mejía

Gabriela Real-Álvarez

Emilia Ortíz-Rodas

Ximena Vélez-Calvo

Universidad de Cuenca - Universidad del Azuay xvelez@uazuay.edu.ec

\section{RESUMEN}

En esta investigación se analizan los conocimientos del profesorado en activo respecto a las características, barreras y facilitadores del alumnado con altas capacidades, a través de un estudio cualitativo realizado las respuestas mediante un ań́lisis categórico pues interestaba conta secr con cierta libertad y apertura percepciones que los docites Los resultados señalan que existe un desconcimiento sobre las Altas Ca-

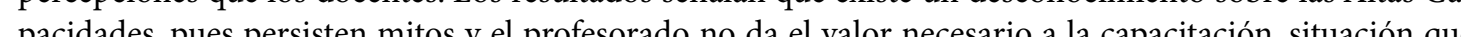
implica que se tomen medidas urgentes para atender a este grupo y a sus necesidades educativas especiales.

Palabras clave: : ALTAS CAPACIDADES, BARRERAS, CARACTERÍSTICAS, FACILITADORES, PROFESORES

\section{ABSTRACT}

Giftedness: what teachers know about these students

This research analyzes the knowledge of teachers regarding characteristics, barriers and facilitators of gifted students, through a qualitative study realized on 30 teachers of basic general education. A semi-structured interview was applied, and the answers were systematically analyzed. These results indicate that myth continue about gifted and the lack of value that professors give to training It is a situation that implies urge actions to focus on this group of people and their special educational needs.

Keywords: BARRIERS, CHARACTERISTICS, FACILITATORS, GIFTED CHILDREN, TEACHERS 
crear una cultura escolar donde se sientan incluidos, valorados y competentes, independientemente de sus intereses capacidades (Fathy y Farahat, 2019). Las principales barreras para conseguir que los sistemas educativos sean verdaderas organizaciones atentas a la diversidad de los estudiantes con $\mathrm{AC}$, son las creencias, normas, ideas y comportamientos de lo agentes implicados en el proceso educativo (Fernández y Benítez, 2016). Estas condiciones previas, han afectado las prácticas educativas y fomentan la marginación y desigualdad de este alumnado (Âlvarez Y Buenestado, 2015).

Los principales facilitadores para el éxito educativo de estos estudiantes son: el rol docente, sus competencias y su mirad hacia la diversidad. Otros facilitadores son la transformación de las metodologías, la reestructuración de las estructuras organizativas y la apertura hacia otros enfoques (Arnaíz y Guirao, 2015). Para garantizar el máximo desarrollo del potencia de su alumnado (Worrell et al., 2019) el docente es quien deben actuar como catalizador ambiental (Gagné, 2015), y los facilitadores pueden ser recursos para logra este reto.

Algunos trabajos han demostrado que los docentes tienen varias confusiones sobre las condiciones y las respuestas que deben generarse para los estudiantes con AC (Del Rosal y Navieras, 2016; Gallardo, 2017; Ovalles, 2018). Muchos profesores consideran que los estudiantes con $\mathrm{AC}$ tienen ciertas características que los hacen únicos. Un ejemplo común es la idea del niño que se destaca en todas las áreas, cuando suele pasar que se muestra profundamente interesado y altamente motivado en ciertos aspectos y descuida lo que no son de su interés (López, Martín y Palomares, 2020).

Los falsos mitos de las AC siguen vigentes y dificultan los procesos educaivos de este colectivo (Tejera, Del Rosa y Navieras, 2017). Entre estos se pueden destacar que los estudiantes con AC siem- pre tienen buenas notas, que no necesitan apoyo en la escuela, que se consideran superiores y que la única medida educativa para estos niños es adelantarlos un curso escolar (Castro, Alarcón y Reguero, 2019; Secanilla, 2019).

Las notorias características diferenciales de este grupo de estudiantes exigen que los sistemas educativos y de manera especial el cuerpo docente, contemplen de manera explícita el abordaje de esta $\mathrm{NE}$ (Palomares y García 2017). En este contexto, el objetivo de esta investigación es determinar que conocen los docentes respecto a las características y a las barrera y facilitadores escolares de los estudiante con AC.

\section{Método}

Debido a la naturaleza exploratoria del estudio, se empleó un método de investigación cualitativa pues interesaba conocer con cierta libertad y apertura las percepciones que los docentes tienen respecto a las características, barreras y facilitadores de los niños con AC (Pedreira, Aguaded y Rodríguez, 2019).

\section{Participantes}

Se entrevistó a 30 profesores de Eduación General Básica de escuelas públicas y privadas de la ciudad de Cuenca-Ecuador. La mayoría de los participantes fueron mujeres $(n=29)$. La media de edad fue $36,4$ años (DT: 3,5$)$

\section{Herramienta}

Utilizamos una entrevista semiestructurada en la que se consultaron los siguientes temas:

- ¿Qué son las AC?

- ¿Cuáles son las características de los niños con $\mathrm{AC}$ ?

- ¿Tienen estos niños necesidades educativas?

- ¿Cuáles son estas necesidades?

- ¿Qué podemos hacer para apoyar el aprendizaje de estos niños?
El concepto de AC ha evolucionado
Diversas propuestas se han realizado para delimitar los indicadores de la educación de los estudiantes con AC y así 


\section{Procedimiento}

Se seleccionaron los participantes mediante el método de bola de nieve (Pedreira et al., 2019). Luego de gestionar los respectivos consentimientos informados, aplicamos y grabamos en audio la entrevista a cada uno de los participantes. A continuación, se procedió a transcribir la información para proceder al respectivo análisis cualitativo.

El análisis de la información se lo realizó con el apoyo del Software NVIVO 12. Se clasificó la información en dos categorías de análisis: 1) Conocimiento de los docentes respecto a las características de las AC, 2) Barreras y facilitadores de lo estudiantes con AC.

Para cada categoría, dos investigadoras clasificaron por separado hasta satura la información a partir de la abreviatura "P" seguida del número de entrevistado. Es decir, el primer maestro entrevistado es tratado como "P1", el segundo como "P2" y así sucesivamente. La Figura 1 present un ejemplo de esta saturación.

Finalmente, se cruzó la información arrojada por el Software con el fin de encontrar las diferencias y llegar a acuerdos.

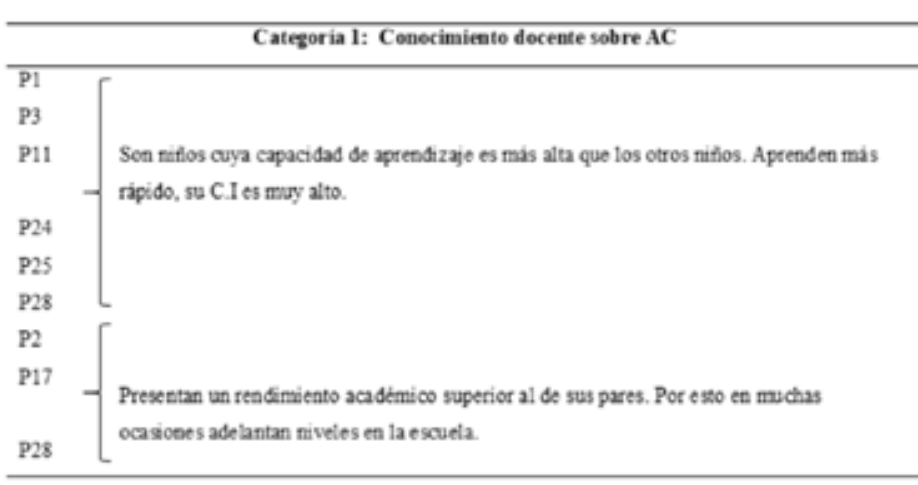

Figura 1. Ejemplo de saturación

\section{Hallazgos}

Los hallazgos se presentan a continuación, organizados en las dos categorías. En un primer momento se mencionará el hallazgo y en un segundo momento, una frase textual de los participantes, que argumenta el por qué del hallazgo

Para la primera categoría, conocimiento de los docentes respecto a las características de las $A C$, se han hallado cinco aspectos relevantes:

- Los niños con AC presentan un rendimiento académico y un C.I muy superior.

Son niños cuya capacidad de aprendizaje es más alta que la de los otros niños. Aprenden más rápido y su C.I es muy alto (P1, P3, P11, P24, P25, P28).

- Las características de los niños con AC pueden variar entre un sujeto otro; son capaces de realizar varias actividades sin ningún tipo de dificultad.

No todos los niños con AC son iguales, sin embargo, todos sí son capaces de realizar varias actividades con mucha facilidad, por ejemplo, tocan varios instrumentos, hablan varios idiomas, resuelven problemas con mucha facilidad, etc. (P8, P9, P17, P21, P21).

- Las altas capacidades son innatas. Las AC no se puede adquirir con el paso del tiempo es algo que ya viene innato en cada persona (P16, P17, P20, P23, P26).

Los niños con AC son muy estudiosos, incluso pueden estudiar simultáneamente varias carreras en la universidad.

No conocemos mucho al respecto, pero lo que hemos escuchado es que estos niños son muy estudiosos, incluso cuando llegan a la universidad son los que pueden estuCiar más de dos carreras (P10, P13, P15, P29).

- Los niños con AC no necesita ningún tipo de apoyo en la escuela.

Estos niños como son tan inteligentes, no necesitan ningun apoyo en la escuela (los pueden todo solos (P2, P3, P12, P15, P20, P27, P30).

Para la segunda categoría, barreras y facilitadores de los estudiantes con $A C$ se han hallado tres aspectos relevantes:
- Los docentes no conocen sobre la $\mathrm{AC}$, situación que muchas de las veces les leva actuar de forma errónea.

No hemos tenido ningún tipo de capacitación formal sobre $A C$, lo que sabemos es lo que buenamente por nuestra propi voluntad hemos aprendido, por eso no sabemos cómo trabajar con estos niños y les apoyamos muy poco (P2, P4, P5, P6, P10 P11, P13...).

- Las AC no debería ser un tema de capacitación docente, porque en nuestro contexto las AC no existen.

En nuestro contexto los niños superdotados no existen, ese no debería ser un tema prioritario de capacitación, tenemo otros problemas más importantes que de berían ser atendidos (P1, P3, P17, P18, P22 P26, P29, P30)

- Los docentes están dispuesto apoyar a sus estudiantes con AC.

Si tuviéramos un alumno con $A C$ le brindaríamos estrategias y el apoyo para que no se aburra en clases, les diéramo otras actividades más complejas, le atenderíamos de mejor manera (P6, P8, P11, P13, P16, P21, P22, P30).

\section{Discusión}

El objetivo de este trabajo fue investiar el conocimiento de docentes respecto a las características, barreras y facilitadore de los niños con AC a través de entrevista semiestructuradas.

Para empezar, una de las características que los docentes consideran como destacadas de los niños con AC es el rendimiento académico superior al de sus pares. Esta afirmación es un mito común e el profesorado, pues hay niños con $\mathrm{AC}$ que pueden mostrar un desempeño escola promedio o bajo, porque pueden no estar interesados en sobresalir en ciertas áreas, pero si se destacan en ámbitos donde su talento emergente puede desarrollarse (Martín-Lobo, Pradas y Navarro, 2018).

Los participantes también manifestaon que las personas con $\mathrm{AC}$ tienen un $\mathrm{CI}$ significativamente superior. No obstante, las AC no pueden valorarse únicamente través de una medida de inteligencia, ni su talento es resultado de las puntuaciones de las pruebas psicométricas (Peyre, Charkaluk, Forhan, Heude y Ramus, 2017). Es necesario, contrarrestar y analizar estos resultados con evaluaciones objetivas y subjetivas (Sánchez y Baena, 2017) y con valoraciones más profundas y complejas, desde diferentes miradas y dimensiones (Pfeiffer, 2017).

La mayoría de los profesores reconocen no tener un concepto claro sobre las AC, pues no han recibido formación inicial o continua respecto al tema, a pesar de que esta condición está reconocida en el Reglamento a la Ley Orgánica de Educación Intercultural desde cia, un gran número de alumnos con $\mathrm{AC}$ pasan desapercibidos en la escuela, pues si el docente no esta preparado, dificilmente podrá detectar a tiempo, las características más notorias de sus estudiantes (López et al., 2020). Por otro lado, muchas de las veces los confunden con trastornos comportamentales y no les dedican la atención necesaria dentro del aula (Luria, O’Brien y Kaufman, 2016).

Los docentes también mencionaron que las $\mathrm{AC}$ no deberían ser tema de formación docente, pues algunos no creen que existan niños con esta condición y hay otras prioridades de capacitación. Una de las razones por las que el profesorado minimiza la existencia de las AC es la falta de información y las creencias erróneas que impiden que estos niños se identifiquen $y$ valoren socialmente (Carlson, HolcombMcCoy y Miller, 2017). Precisamente, si hay niños con AC y en esta ciudad se encontró una prevalencia del $6,2 \%$ en la población escolar (Vélez-Calvo et al., 2019).

Los participantes señalaron que las características de las AC pueden variar entre un sujeto y otro. Efectivamente, investigaciones manifiestan que algunos niños con AC pueden desarrollar sus capacidades de diversas maneras y antes que los niños de su edad. Sin embargo, otros sujetos pueden desarrollarse al mismo tiempo 
que sus pares, sobre todo aquellos que no logran destacar demasiado, debido a que se mimetizan con su entorno (Callahan, 2017).

Los profesores también señalaron que los niños con AC son capaces de realiza varias actividades sin ningún tipo de dificultad, por ejemplo, tocan varios instrumentos, aprenden a hablar rápidamente varios idiomas, resuelven problemas con mucha facilidad, etc. En efecto alguno niños con AC muestran respuestas notablemente elevadas, o el potencial necesario para alcanzarlas, comparados con lo demás individuos de su misma edad. Sin embargo, si estos estudiantes no reciben la motivación y estimulación necesaria, es muy probable que su potencial/talento disminuya de manera notoria; o incluso ese talento desaparezca (Tourón, 2020) Por otro lado, su talento podría no demostrarse hasta que no encuentre el impulso que necesita para desarrollarse o que esto niños con talentos emergentes también requieran respuestas que atiendan sus necesidades educativas específicas (Secanilla 2019).

Los docentes participantes manifestaron que las $\mathrm{AC}$ no se pueden adquirir con el paso del tiempo, pues son innatas a cada persona. Investigaciones han confirmado que la herencia juega un papel muy importante en la presencia de las $\mathrm{AC}$ pero por sí solas no emergen (Herranz y Sánchez, 2019). Es necesario la presenci de catalizadores que activen los potenciales intelectuales, pues de esta manera, el resultado final será producto de la interacción entre el aprendizaje, la herencia otras variables que influyen en el proceso (Pace, 2019).

Los participantes también mencionaron que los niños con $\mathrm{AC}$ pueden ser muy estudiosos, inclusive cursar simultáneamente dos o tres carreras en la universidad. Al hacer una revisión teórica, ningún estudio asegura lo manifestado por lo participantes, sin embargo, es habitual que los docentes piensen de esta manera (López et al., 2020), siendo este uno de los mitos más comunes (Mendioroz, Rivero y Aguilera, 2019)

Un gran porcentaje de los docen tes coincidieron en que los niños con $\mathrm{AC}$ no necesitan ningún tipo de apoyo, pues como son tan inteligentes no requieren ayudas dentro del salón de clase. No obstante, estos niños demandan apoyos específicos que les permitan alcanzar su desarrollo intelectual y personal (Herranz Sánchez, 2019). Ignorar estas necesidades puede llevarnos a cometer errores como: frenar su curiosidad, sus ganas de aprender cosas nuevas, apagar su motivación y llevarlos a esforzarse lo menos posible, situaciones que pueden traer entre otros problemas el fracaso escolar (Heilat y Seifert, 2019; Jovanovic y Vukić, 2019).

Finalmente, los participantes, también manifestaron que están dispuestos apoyar a sus estudiantes con AC. Este es un facilitador que puede tener un efecto considerable en el desarrollo positivo de estos chicos (González, Trillo y Goig 2019). Sin embargo, se debe tener en cuenta que un profesor tendrá mayor intención de cambiar sus prácticas, cuanto mejo sea su actitud y disponibilidad, pese a la barreras que deba superar (Magro, 2019). Para ello, deberá sentirse competente en el manejo de estos procesos escolares, además debe estar formado en aspectos relacionados con la atención a la diversidad y de sentirse apoyado con las demandas organizativas que implica su propia gestión educativa (González et al., 2019).

\section{Conclusiones}

En este estudio se pudo evidenciar que los profesores tienen muchas dudas falsas creencias respecto a las AC. Las características que usaron los maestros para describir a estos niños caen en el terreno de los mitos que se buscan superar: tiene buen rendimiento académico, son autosuficientes, no necesitan apoyo, pueden estudiar varios temas y realizar varias actividades, su talento es innato y se desarrolla sin esfuerzo. Además, todos los profesores conciben a estos niños con un perfil marcadamente académico, cuando estos chicos pueden tener potencial para las artes, el deporte, las ciencias y el liderazgo, lo que al parecer no son tan importantes como ser buenos alumnos.

Por otro lado, las barreras más preocupantes son que los profesores no conozcan sobre las AC y a su vez, no sientan necesidad de capacitarse. Estas percepciones se convierten en un círculo vicioso, pues el desconocimiento genera comodidad y esta comodidad una pasividad que alimenta el desconocimiento.

\section{Referencias bibliográficas}

Álvarez, J.y y Buenestado, M. (2015). Predictores de las actitudes hacia la inclusión de alumnado con necesidades educativas especiales en futuros profesionales de la educacion. Revista Compluten de Educacion, 26(3), 627-645.

Arnáiz, P., y Guirao, J. (2015). La autoevaluación de centros en España para la atención a la diversidad desde una perspectiva inclusiva: ACADI. Revist Profesorado, 18(1), 45-101.

Azorín, M., Arnaiz, P., y Maquilón, J. (2017). Revisión de instrumentos sobre atención a la diversidad para una educación inclusiva de calidad. Revista mexicana de investigación educativa, 22(75) 1021-1045.

Barrenetxea-Mínguez, L., y Martínez-Izaguirre, M. (2019). Relevancia de la formación docente para la inclusión educativa del alumnado con altas capacidades intelectuales. Atenas, 1(49), 1-19.

Callahan, C. (2017). The Characteristics of Gifted an Talented Students. Londres-Inglaterra: Routled-

ge.
Carlson, N., Holcomb-McCoy, C., y Miller, T. (2017). School counselors' knowledge and involvement concerning gifted and talented students. Journa of Counselor Leadership and Advocacy 4(2) 89101.

Castro, S, Alarcón, E., y Reguero, M. (2019). - Tienen mitos los estudiantes de Magisterio sobre alumnado con Altas Capacidades Intelectuales? Revista Riuma, 1(9), 19-28.

Comes, G., Díaz, E., De la Rosa, A, y Moliner, O. (2016). La evaluación psicopedagógica del alumnado con altas capacidades intelectuales. Revista d Educación Inclusiva, 1(1),345-360.
Es necesario resolver estos mitos $y$ barreras por medio de una buena preparación docente, inicial y continua, y que e sistema educativo asuma con mayor responsabilidad la educación de este grupo desde los diferentes niveles, agentes e instancias que lo componen.

Limitaciones y estudios futuros

Este estudio cualitativo no permite la generalización de los resultados, pero si un puede ser un valioso punto de partida para realizar trabajos de mayor alcance con profesores de otras regiones y de diferentes niveles a partir de las problemáticas que este trabajo declara. $\infty$

Del Rosal, B., y Navieras, E.. (2016). Superdotación y altas capacidades intelectuales, tierra de mito RIDPSICLO, 1(3), 11-20.

Fathy, M., y Farahat, A. (2019). Effectiveness of a Model Based on the Developmental Cognitive Theory International Journal for Talent Development, 10(19), 135-164.

Fernández, E., García, T., Arias-Gundín, O., Vázquez A., y Rodríguez, C. (2017). Identifying gifted children: Congruence among different IQ measures. Frontiers in psychology, 8(1), 1-10.

Fernández, J., y Benítez, M. (2016). Respuesta educativa de los centros escolares ante alumnado con Síndrome de Down: percepciones familiares docentes. Profesorado. Revista de curriculum
formación del profesorado, 20(2), 296-311. né, $F$. (2015). De los genes al talento: la perspectiva DMGT/CMTD. Revista de Educación, 6(368), 12-39.

Gallardo, R. (2017). El Aprendizaje-Servicio como una estrategia inclusiva para superar las barreras al aprendizaje ya la participación. Revista de Educación Inclusiva, 5(1), 71-82.

García, A., y De la Flor, P. (2016). Percepción del profesorado español sobre el alumnado con altas capacidades. Estudios pedagógicos Valdivia, 42(2), 129-149.

García, R. (2017). Acercamiento a la realidad de las alta capacidades en España: prevalencia y variables moduladoras. V seminário internacional cognição, aprendizagem e desempenho, 1(1), 6-19.

García, R., y García, A. (2016). Conocimientos y pecepciones docentes sobre la respuesta educativa a los alumnos con altas capacidades intelectuales. Livro de Atas, 1(1), 36-48.

Gonzalez, M., Trillo, M., y Goig, R. (2019). Atención a la Diversidad y Pedagogía Diferencial. Madrid - 
España: UNED.

Henao, J. (2019). Diseño Curricular para la atenció educativa de jóvenes con talentos y con Capacidades Excepcionales en Instituciones Inclusivas.

Heilat, M., y Seifert, T. (2019). Mental motivation, intrinsic motivation and their relationship wit emotional support sources among gifted and non-gitted Jordanian adolescents. Cogent

Herranz, G., y Sánchez,A. (2019). Tratamiento de las als capacidades en el sistema educativo españo: (1) 11(1), $93-110$.

Higueras-Rodríguez, L. (2017). Intervención educativa en el alumnado con altas capacidades. Revista , 69-81.

Jovanovic, M., y Vukić, T. (2019). Types of support gifted students receive in school. Facta Universitatis, Series: Teaching, Learning and Teacher Edu-

López, E., Martín, M., y Palomares, A. (2020). Empoderamiento docente en el ámbito de las altas capacidades intelectuales. Mitos y crencias en los docentes de

Luria, S., O'Brien, R., y Kaufman, C. (2016). Creativity in gifted identification: Increasing accuracy and diversity. Annals of the new York Academy of

Magro, M. (2019). Necesidades formativas del profesorado de educación infantil para la atención a la diversidad de colegios rurales agrupados. Revista Martin-Lobo, P. Pradas S, y Navat o

dio de la Aplicación de Programas (2018). Estudimiento para alumnos con altas Capacidades. Electronic Journal of Research in Education Psychology, 16(45), 447-476.

Mendioroz, A., Rivero, P., y Aguilera, E. (2019). Un propuesta de formación docente para responder a las altas capacidades en la escuela inclusiva Profesorado, Revista de Currículum y Formación del Profesorado, 23(1), 265-284.

Ovalles, A. (2018). Necesidades de Capacitación de los Docentes de Educación Inicial sobre las Altas Capacidades Intelectuales. Revista Scientific, $3(10), 20-39$.

Pace, J. (2019). Giftedness in Childhood. The Encyclopedia of Child and Adolescent Development $1(1), 1-12$.

Palomares, A., y García, R. (2017). Innovación y creatividad para favorecer la intervención educativa del alumnado con altas capacidades. Revista de Educación Inclusiva, 9(1), 90-100.

Pedreira, M., Aguaded, I., y Rodríguez, M. (2019). Educomunicación y Buenas Prácticas en los nuevos arios tecnológicos: Estudio de Caso. $\mathrm{H}$ mutay, 6(1), 96-111.

eyre, H., Charkaluk, M., Forhan, A., Heude, B., y Ramus, F. (2017). Do developmental milestones a 4, 8, 12 and 24 months predict IQ at 5-6 years European journal of paediatric nerold cohrt. 272-279.

Pfeiffer, S. (2013). Serving the gifted: Evidence-based clinical and psychoeducational practice. New Yrork

. Focusing on strengths of the head and strengths (3)

Ranz, R., y Tourón, J. (2017). Características del alumnado con altas capacidades, algunas pitas para su

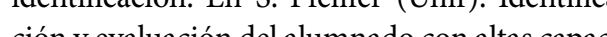

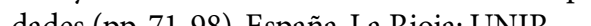
distro Oficial (2012). Ley Orgánica de Educa-

ción Intercultural. Recuperado de de Educa-

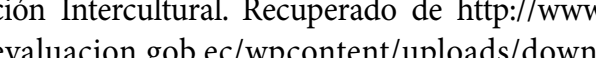
/ lo

ción del alumno con altas). Identificaintelectuales:-responsabilidad del maestro o de departamento de orientación educativa y psicopedagógica? Aula de Encuentro, 19(1), 69-91. Seade, C., Encalada G. Peñaherrera-Vélez, M. Dávila Y., y Vélez-Calvo, X. (2019). ¿ Puede la formación en altas capacidades afectar las actitudes de los maestros en educación primaria? Revista IN FAD de Psicología, 5(1), 441-450.

Secanilla, E. (2019). Supermentes: Reconocer las altas capacidades en la infancia. Barcelona - España GEDISA.

Subotnik, R., Olszewski-Kubilius, P., y Worrell,F. (2011). Rethinking giftedness and gifted education: $A$ proposed direction forward based on psychological science. Psychological Science in the Public Interest, 12(1), 3-54.

Tejera, J., Del Rosal, B., y Naveiras, E. (2017). Conocimientos y Mitos sobre Altas Capacidades. Talincrea: Revista Talento, Inteligencia y Creatividad 4(1), 40-51.

Tourón, J. (2020). Las Altas Capacidades en el sistema educativo español: reflexiones sobre el concepto la identificación. Revista de Investigación Educativa, 38(1), 15-32.

Vélez-Calvo, X., Dávila, Y., Seade, C., Cordero, M., y Peñaherrera-Vélez, M. (2019). Las altas capacidades en la educación primaria, estudio de prevalencia con niños ecuatorianos. Revista INFAD de Psicología, 5(1), 391-400.

Worrell, F., Subotnik, R., Olszewski, P., y Dixson D. (2019). Gifted students. Annual review of psychology, 70(2), 551-576.

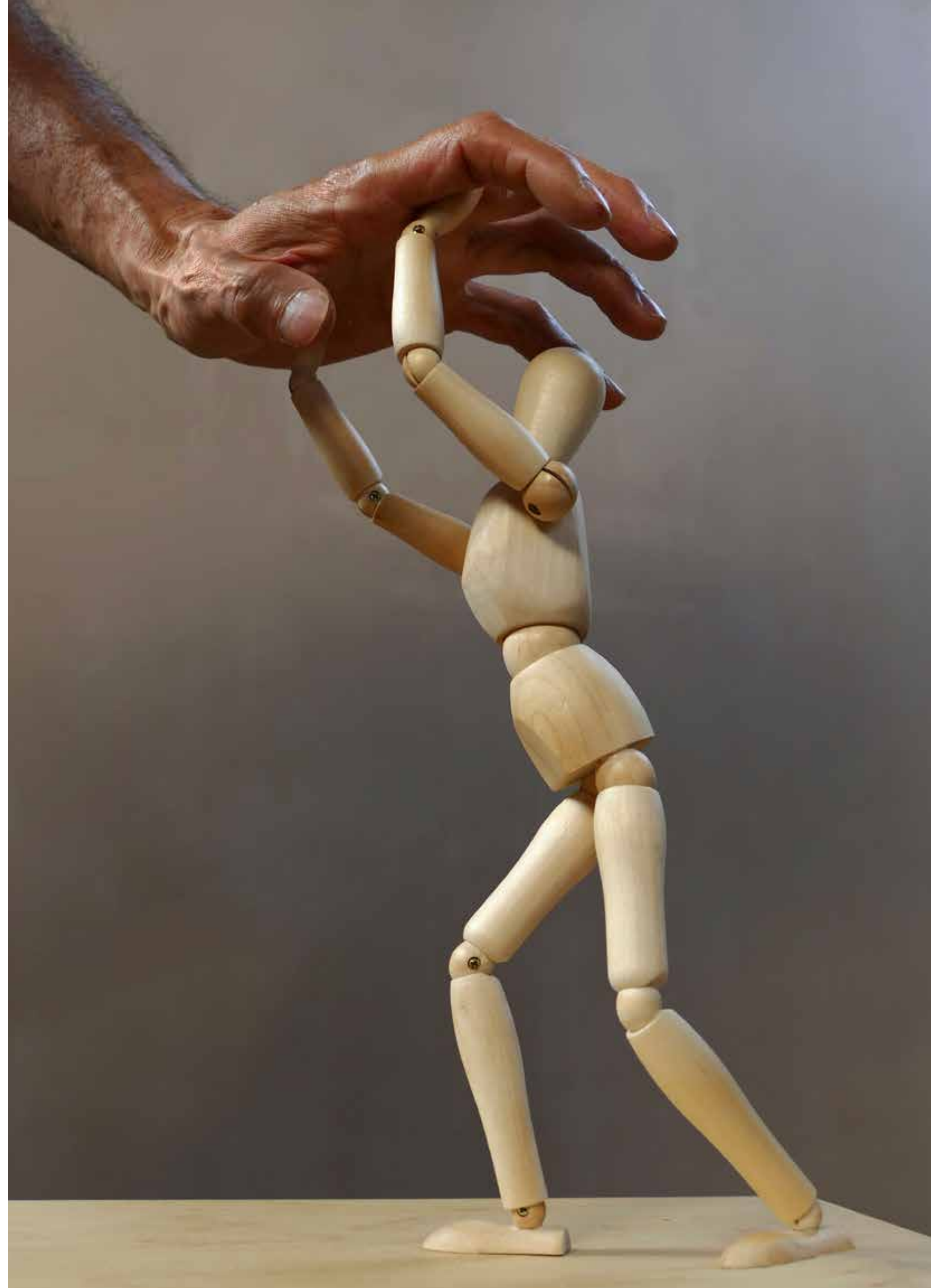

